\title{
A Mechanistic Model for Reward Prediction and Extinction Learning in the Fruit Fly
}

\author{
Magdalena Springer and ${ }^{\circledR}$ Martin Paul Nawrot
}

https://doi.org/10.1523/ENEURO.0549-20.2021

Computational Systems Neuroscience, Institute of Zoology, University of Cologne, Biocenter, Cologne 50674, Germany

\section{Visual Abstract}

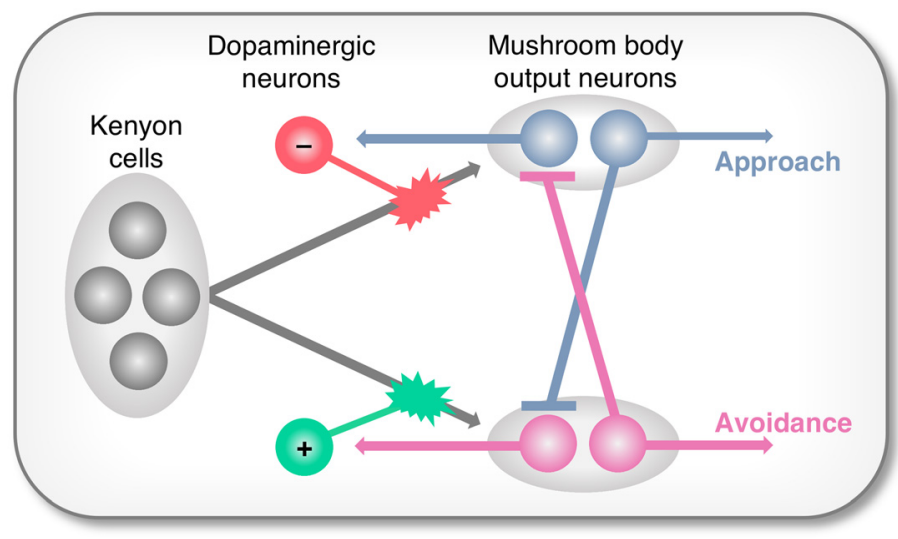

Appetitive Learning

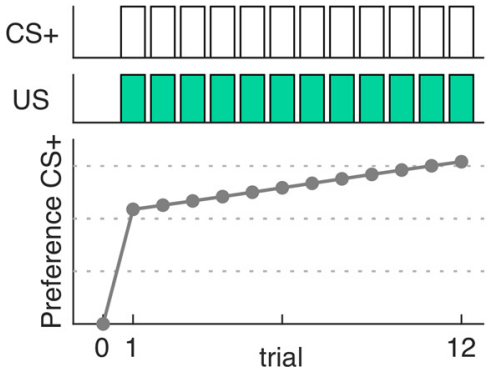

Extinction Learning

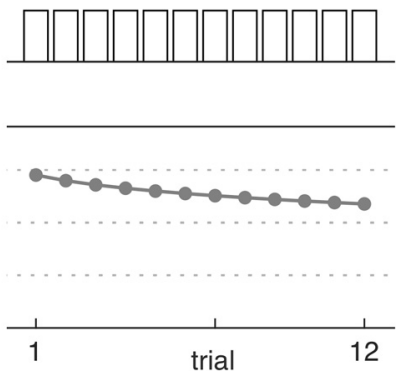

\section{Significance Statement}

A stressful experience can lead to a strong fear memory where a negative consequence has been associated with a certain stimulus or event. This can trigger fear whenever the same or similar event occurs. Extinction of such a maladaptive memory through extinction learning can thus be of high therapeutic value. Here, we present novel theoretical work on the formation and extinction of memories in the fruit fly that suggests an underlying neural circuit mechanism for reward prediction based on recently reported anatomic, physiological and behavioral data. Our findings propose how the theoretical concept of prediction error coding can be realized in a biologically realistic neuronal circuit motif to enable associative learning, saturation of learning, single-trial memory, and memory extinction. 
Extinction learning, the ability to update previously learned information by integrating novel contradictory information, is of high clinical relevance for therapeutic approaches to the modulation of maladaptive memories. Insect models have been instrumental in uncovering fundamental processes of memory formation and memory update. Recent experimental results in Drosophila melanogaster suggest that, after the behavioral extinction of a memory, two parallel but opposing memory traces coexist, residing at different sites within the mushroom body (MB). Here, we propose a minimalistic circuit model of the Drosophila MB that supports classical appetitive and aversive conditioning and memory extinction. The model is tailored to the existing anatomic data and involves two circuit motives of central functional importance. It employs plastic synaptic connections between Kenyon cells (KCs) and MB output neurons (MBONs) in separate and mutually inhibiting appetitive and aversive learning pathways. Recurrent modulation of plasticity through projections from MBONs to reinforcementmediating dopaminergic neurons (DAN) implements a simple reward prediction mechanism. A distinct set of four MBONs encodes odor valence and predicts behavioral model output. Subjecting our model to learning and extinction protocols reproduced experimental results from recent behavioral and imaging studies. Simulating the experimental blocking of synaptic output of individual neurons or neuron groups in the model circuit confirmed experimental results and allowed formulation of testable predictions. In the temporal domain, our model achieves rapid learning with a step-like increase in the encoded odor value after a single pairing of the conditioned stimulus (CS) with a reward or punishment, facilitating single-trial learning.

Key words: Drosophila melanogaster; memory extinction; reinforcement learning; reward prediction; single-trial learning

\section{Introduction}

Fruit flies can learn to associate an odor stimulus with a positive or negative consequence, e.g., food reward or electric shock punishment. In the training phase flies are typically exposed to two odors (differential conditioning) where one odor [conditioned stimulus minus (CS-)] is perceived alone whereas a second odor [CS plus (CS+)] is presented together with either reward or punishment [unconditioned stimulus (US)]. Once an association has formed between the CS+ and the respective US, the learned anticipation of the US can be observed in a memory test that enforces a binary choice behavior between the CS+ and the CS- (Tempel et al., 1983; Tully and Quinn, 1985). A single learning trial can be sufficient to form a stable memory in the fruit fly (Beck et al., 2000;

Received December 18, 2020; accepted March 18, 2021; First published March 30, 2021.

The authors declare no competing financial interests.

Author contributions: M.S. and M.P.N. designed research; M.S. and M.P.N. performed research; M.S. and M.P.N. analyzed data; M.S. and M.P.N. wrote the paper.

This work was funded by the German Research Foundation in parts within the Research Unit Structure, Plasticity and Behavioral Function of the Drosophila mushroom body (DFG-FOR 2705, Grant 403329959) and within the priority program Evolutionary Optimization of Neuronal Processing (DFG-SPP 2205, Grant 430592330).

Acknowledgements: We thank Johannes Felsenberg, who has been supporting this project throughout with his expertise and fruitful ideas. We also thank Anna-Maria Jürgensen for her contribution to the initial model implementation as well as Bertram Gerber and Anna-Maria Jürgensen for insightful comments on an earlier version of this manuscript.

Correspondence should be addressed to Magdalena Springer at magdalena.springer@uni-koeln.de or Martin Paul Nawrot at martin. nawrot@uni-koeln.de.

https://doi.org/10.1523/ENEURO.0549-20.2021

Copyright (C) 2021 Springer and Nawrot

This is an open-access article distributed under the terms of the Creative Commons Attribution 4.0 International license, which permits unrestricted use, distribution and reproduction in any medium provided that the original work is properly attributed.
Krashes and Waddell, 2008; Zhao et al., 2019) and other insect species (see Discussion).

The prediction error theory (Rescorla and Wagner, 1972) describes a basic theoretical concept of classical conditioning. It assumes that the efficacy of learning is determined by the momentary discrepancy (or error) between the expected and the received reinforcement (i.e., reward or punishment). In vertebrates, it has been shown that prediction error coding dopaminergic neurons (DANs) are involved in learning (Schultz et al., 1997; Schultz, 2016). Recent studies give rise to the assumption that DANs could play a similar role in Drosophila melanogaster (Hammer, 1997; Riemensperger et al., 2005; Ichinose et al., 2015; Eichler et al., 2017; Felsenberg et al., 2017, 2018; Zhao et al., 2021; Eschbach et al., 2020) and other insects (Terao and Mizunami, 2017), rejuvenating an earlier hypothesis based on experimental observations in the honeybee (Hammer, 1997).

Re-exposing the flies to the CS+ after successful training and in the absence of the US leads to a reduction of the previously learned behavior (Tempel et al., 1983; Schwaerzel et al., 2002; Felsenberg et al., 2017, 2018). This new learning is called extinction learning and has been observed across invertebrate (Eisenhardt and Menzel, 2007; Eisenhardt, 2014) and vertebrate species (Pavlov, 1927; Tully and Quinn, 1985; Myers and Davis, 2002; Bouton, 2004, 2017; Dudai, 2004). Following prediction error theory, extinction learning is caused by the mismatch between the expected outcome (predicted US) based on the initial learning and the actual outcome (no US). In humans, extinction learning is of high clinical relevance and is applied in the treatment of humans suffering from maladaptive memories (Quirk and Mueller, 2008; Chiamulera et al., 2014; Delamater and Westbrook, 2014; Bouton, 2017; Walsh et al., 2018). The majority of behavioral experiments in invertebrates and vertebrates suggest that extinction protocols, including the applied exposure therapy in humans, does not erase a memory 
but rather leads to the formation of a parallel but opposing memory, the extinction memory (Bouton, 2004; Eisenhardt and Menzel, 2007). Despite the relevance for clinical therapy, our understanding of the neural circuit mechanisms underlying extinction learning is still in its infancy.

We study a computational neural circuit model of the fruit fly mushroom body (MB) that captures the most recent anatomic and physiological facts. The model proposes detailed mechanisms for associative learning, extinction learning, prediction error coding, and singletrial learning. We compare the model outcomes quantitatively to the results of recent behavioral and physiological studies and we derive novel experimental predictions by mimicking neurogenetic manipulations of relevant neuron groups.

\section{Materials and Methods}

\section{Circuit architecture}

Our circuit model for olfactory coding and olfactory memory formation (Fig. 1) consists of three neuron layers [projection neurons (PNs), Kenyon cells (KCs), MB output neurons (MBONs)] representing the three major stages of the olfactory pathway in Drosophila and two reinforcement mediating DANs representing the PAM and PPL1 cluster. Each neuron in the circuit can assume an activation rate in the range of $0-1$.

Olfactory model input was simulated through the activation of 50 out of the total 100 PNs. This matches experimental observations of $\sim 40-60 \%$ of PNs being activated by a single odor stimulus (Krofczik et al., 2009; Brill et al., 2013; Wilson, 2013) and follows the model of Peng and Chittka (2017). Each PN is activated with a random rate drawn from a uniform distribution in the range between 0.2 and 0.8 . To enhance variability between the networks that were set up, PN input patterns were further multiplied by a factor that was randomly distributed between 0.8 and 1 . PNs are connected to $2000 \mathrm{KCs}$ via the weight matrix W1 where each PN is connected to $5-15 \mathrm{KCs}$, each connection has a fixed synaptic weight of 0.2 . Activation of the $\mathrm{KC}$ vector in the next layer resulted from the matrix product of the PN population vector and the respective weight matrix W1 according to

$$
K C=P N \times W 1 .
$$

Out of the total $2000 \mathrm{KCs}$ only the subpopulation of 100 KCs $(5 \%)$ with the highest activation rate kept its activation. All other KCs are set to zero to enforce population sparseness (Peng and Chittka, 2017). In a next step, KCs are fully connected to the four MBONs via the weight matrix W2, with all synaptic weights initially set to 0.01 . The excitatory input to each MBON was calculated as the matrix products

$$
\begin{gathered}
M 6^{+}, M V 2=K C \times W 2_{a v} \\
M V P 2, V 2^{+}=K C \times W 2_{a p p} .
\end{gathered}
$$

We further included lateral connectivity between the MBONs. The M6 MBON receives inhibitory input from

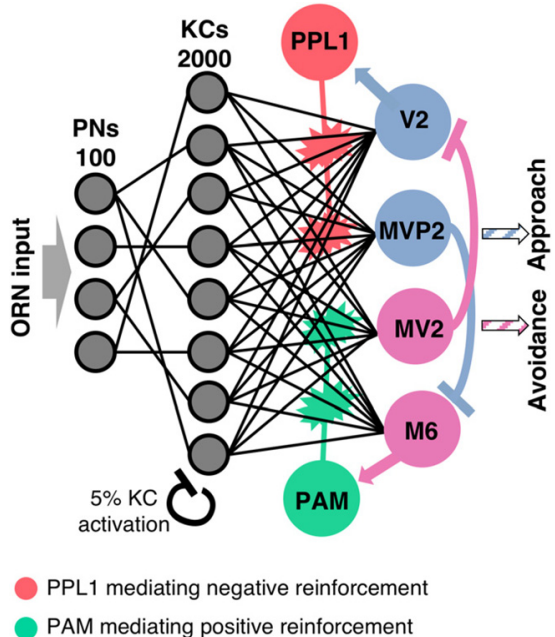

Figure 1. Circuit model. The olfactory pathway comprises three feedforward layers. Olfactory input activates an odor-specific combination of PNs. The connectivity matrix from PNs to the large population of KCs is divergent-convergent and sparse. The inhibitory feedback mechanism ensures population sparseness of $5 \%$ active cells in the KC layer. KCs are fully connected with four MBONs in the output layer. A positive or negative reinforcement stimulus directly excites the dopaminergic PAM or PPL1 neuron, respectively. Lateral inhibition between the MBONs and excitatory feedback from MBONs to DANs is crucial for the reward prediction and extinction mechanisms. Activation of the MVP2 output neuron mediates approach behavior, activation of MV2 mediates avoidance behavior. Behavioral preference toward an odor is calculated as the imbalance between the activations of MVP2 and MV2.

MVP2, whereas V2 gets inhibited by MV2. The respective inhibitory inputs are formalized according to

$$
\begin{aligned}
& M 6^{-}=\frac{-0.6}{\left(1+200 \times e^{(-M V P 2 \times 15)}\right)} \\
& V 2^{-}=\frac{-0.6}{\left(1+200 \times e^{(-M V 2 \times 15)}\right)} .
\end{aligned}
$$

The activation rate of $\mathrm{M} 6$ and $\mathrm{MV} 2$ results from a summation of inhibitory and excitatory input as

$$
\begin{aligned}
& M 6=M 6^{+}+M 6^{-} \\
& V 2=V 2^{+}+V 2^{-},
\end{aligned}
$$

whereas for MVP2 and MV2, the activation rate is solely determined by the excitatory input.

The PAM and PPL1 DAN receive excitatory input by the M6 and V2 neurons, respectively. Additionally, reinforcing stimuli have an effect on both DANs. A rewarding US (US $=+1$, positive reinforcer) leads to an excitatory input to the PAM (Eq. 5a). At the same time, excitatory input from the V2 to the PPL1 is partially suppressed (represented by the factor $\rho=0.8$ in Eq. $5 b$ ). Conversely, a punishing US (US $=-1$, negative reinforcer) results in excitation of the PPL1 (Eq. $5 b$ ) and in the suppression of excitatory input from M6 to the 
PAM (Eq. 5a). The total DAN input was thus computed as

$$
\begin{aligned}
& P A M_{l n}=\left\{\begin{array}{c}
R+M 6, \text { if US }=+1(\text { reward }) \\
\rho \times M 6, \text { if } \cup S=-1(\text { punishment }) \\
\text { M6, otherwise }
\end{array}\right. \\
& P P L 1_{\text {In }}=\left\{\begin{array}{c}
R+V 2, \text { if } U S=-1 \text { (punishment }) \\
\rho \times V 2, \text { if } U S=+1(\text { reward }) \\
\text { V2, otherwise }
\end{array} .\right.
\end{aligned}
$$

We fixed the US induced excitation to $R=0.3$ for all our experiments. However, generally the parameter $\mathrm{R}$ allows for a modeling of variable reward magnitudes linked to the US such as, e.g., different levels of sugar concentration contained in a rewarding US.

The output activation rate of each DAN encoding the prediction error is calculated with a sigmoid transfer function

$$
\begin{aligned}
P A M & =\frac{1}{\left(1+10000 \times e^{-P A M_{\text {lnout }} \times 19}\right)} \\
P P L 1 & =\frac{1}{\left(1+10000 \times e^{-P P L 1_{\text {lnput }} \times 19}\right)} .
\end{aligned}
$$

\section{Plasticity rule at $\mathrm{KC}:$ MBON synapses}

We implemented synaptic plasticity at the KC::MBON synapses. In the initial state of the model, the weights of all KC:: MBON synapses are set to the same fixed value of 0.01 . Each of the synaptic weights in W2 is subject to synaptic plasticity. At the end of a given trial $t$ and for any synapse

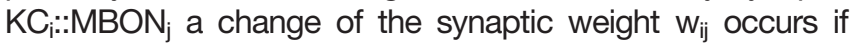
both, the presynaptic $\mathrm{KC}$ and the respective DAN were active in trial $t$. If both conditions are met, the synaptic weight is reduced according to the two-factor learning rule

$$
\begin{gathered}
w_{a p p}=\left\{\begin{array}{c}
w_{a p p}-(P P L 1 \times \delta), \text { if } P P L 1>0, K C>0 \\
0, \text { if } w_{a p p}<0 \\
w_{a p p}, \text { otherwise }
\end{array}\right. \\
w_{a v}=\left\{\begin{array}{c}
w_{a v}-(P A M \times \delta), \text { if } P A M>0, K C>0 \\
0, \text { if } w_{a v}<0 \\
w_{a v}, \text { otherwise }
\end{array}\right.
\end{gathered}
$$

where $\mathrm{w}_{\mathrm{app}}$ refers to the weight of a synapse onto $\mathrm{M} 6$ or MV2 and $w_{a v}$ refers to the weight of a synapse onto MVP2 or V2. Note that the weight changes are proportional to the activation rate of the respective DAN. We fixed the learning rate as $\delta=0.0045$ for all our experiments.

\section{Experimental protocols}

We subjected our model to a set of conditioning and extinction protocols. Further, it was operated and evaluated in a trial-resolved fashion where, in each trial, the excitatory and inhibitory synaptic input to each of the neurons and its output activation rate was computed. Within-trial neuronal dynamics were neglected.

\section{Classical conditioning}

The training procedure derives from a group assay developed by Tully and Quinn (1985) that is often used to study olfactory learning in flies. A group of flies are trained in a Tully-machine with a training arm and two testing arms. In the training arm, the animals are typically exposed to the CS + in combination with either sugar (reward) or a train of electric shocks (punishment). In Felsenberg et al. (2018) the CS+ is presented for $1 \mathrm{~min}$ and combined with 12 electric shocks. Subsequently, animals are exposed to the CS- without reinforcer. After the training, the flies are transferred to the testing chamber in which they can choose between a CS+ and a CS- perfused arm.

Our classical conditioning protocol also consisted of a training phase and a subsequent memory retention test (Fig. $2 A)$. An odor pattern (CS+) was paired with a positive or negative reinforcement (US) during each training trial. Subsequently, a second odor pattern (CS-) was presented without reinforcement. For our initial model analysis, we varied the number of training trials in the range of $n=1, \ldots, 24$ to quantify the associative strength as a function of $n$ (Fig. 2). Then, we fixed the number of training trials to $n=12$ in our standard training procedure. The trial-based classical conditioning protocol is a standard procedure in other insect models for learning and memory, notably the honeybee (Bitterman et al., 1983; Pamir et al., 2011). Unlike in the experimental conditioning paradigm, paired and unpaired odors were presented alternately when the number of trials was $>1$. However, we expect no difference between continuous and alternating odor presentation, since CS- presentation does not induce synaptic plasticity. During retention, we presented the CS+ and CS- stimulus once. In contrast to the experimental paradigm, simultaneous presentation of both odors was not possible. However, effects on the behavioral outcome could be excluded by switching off learning during the retention test.

\section{Extinction learning}

The extinction protocol included an additional reactivation phase after training and before memory extinction (Figure $3 A$ ). During reactivation, the $\mathrm{CS}+$ odor was presented alone, i.e., without reinforcement, during 12 reactivation trials. This procedure again mimics the experimental protocol used by Felsenberg et al. (2018), in which the authors presented the CS + without shocks for the same duration (1 $\mathrm{min}$ ) as in the training.

\section{Neurogenetic manipulation experiments}

To investigate the role of individual neurons in the memory extinction process we selectively suppressed their activation by setting their activation rate to zero during the reactivation phase. Thereby, we mimic neurogenetic manipulation experiments that suppress the activation of specific neurons. We specifically compare our model results to the experimental results performed in Felsenberg et al. (2018) based on the neurogenetic tool shibire ${ }^{\text {ts1 }}$ and we generate experimentally testable hypotheses in novel model experiments. 
A
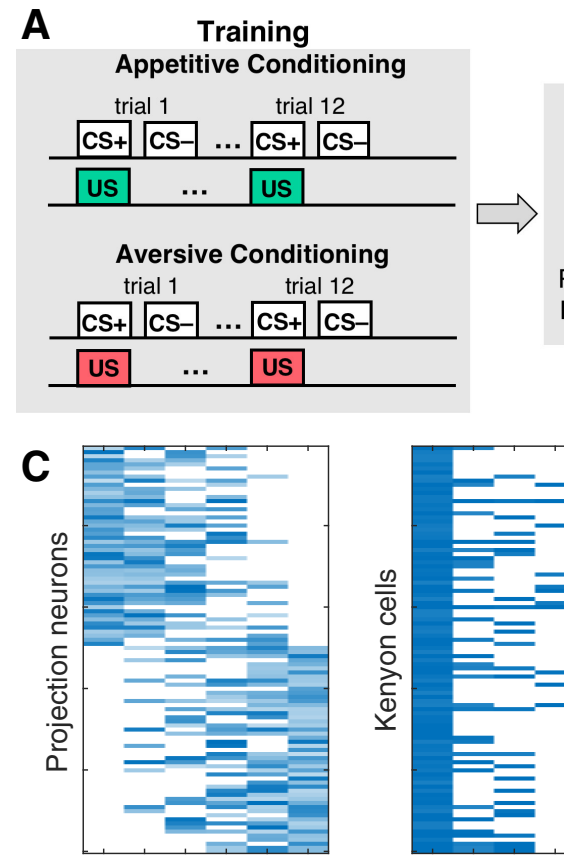

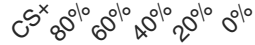

PN pattern overlap
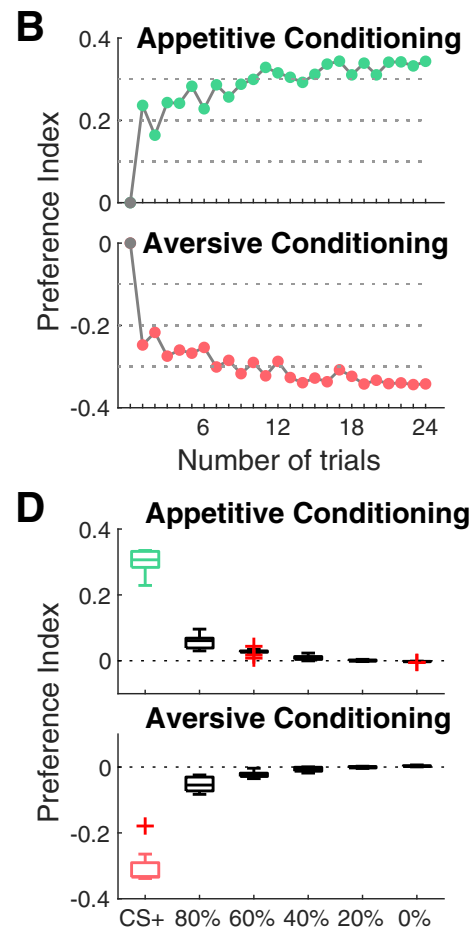

PN pattern overlap

Figure 2. Differential conditioning leads to CS+-specific odor preference. A, Experimental protocols for simulation of associative conditioning experiments. The model was trained in a classical conditioning paradigm, in which a conditioned odor stimulus (CS+) was paired with a reward (green) or punishment (red). Subsequently, a second unconditioned odor stimulus (CS-) was presented without reinforcement. The standard protocol comprises 12 training trials. During the retention test, both odor stimuli were presented alone without reinforcement. Preference index and performance index in the model were computed after each trial and during the retention test. $\boldsymbol{B}$, Dynamics of the odor preference index across 24 appetitive (top) and aversive (bottom) learning trials, respectively averaged across 10 networks. C, left, Combinatorial response patterns in the PN population. Each odor stimulus activates $50 \%$ of all PNs. Similarities between the CS+ reference odor and five novel odors are defined by their overlap in the PN activation pattern ( $x$-axis). Right, Activation patterns across the subpopulation of $100 \mathrm{KCs}(5 \%)$ activated by the CS+ odor. Pattern overlap in the KC population reduces rapidly with decreasing odor similarity as expressed in the percentage of PN pattern overlap ( $x$-axis). $\boldsymbol{D}$, Generalization to different odors after associative conditioning to the CS+ odor. The model shows a significant CS + approach (green) or avoidance (red) after 12 training trials as expressed in the preference index $(n=15)$, which diminishes rapidly with decreasing odor similarity. Boxplots show the median and the lower and upper quartiles, whiskers indicate 1.5 times interquartile range, outliers are marked with + symbol.

\section{Evaluation of model performance and quantitative model predictions}

We evaluated model outcome by different measures that allow for a quantitative comparison with the outcome of animal experiments, both at the behavioral and at the physiological level. Applying these measures in untested experiments allowed us to formulate experimental predictions for novel experiments.

\section{Behavioral output}

To this end, we computed two quantities that can be interpreted as measures of approach or avoidance behavior. We used the activation rates of these MVP2 and MV2 to calculate the preference index as

$$
\text { preference index }=\frac{\left(M B O N_{M V P 2}-M B O N_{M V 2}\right)}{\left(M B O N_{M V P 2}+M B O N_{M V 2}\right)} .
$$

The innate preference index of our model is zero by construction.

In behavioral extinction learning experiments (Felsenberg et al., 2017, 2018), the number of animals that chose the CS- odor was then subtracted from the number of animals that chose the CS+, and the result was normalized by the total group size to compute the performance index (Tully and Quinn, 1985). In analogy, we calculated a model performance index computing the difference between the preference indices for CS+ and CS- as

$$
\begin{aligned}
\text { performance index }= & \text { preference } i n d e x_{C S+} \\
& - \text { preference } i n d e x_{C S-} .
\end{aligned}
$$

\section{Dendritic input}

We quantified activation rates of single neurons. Activation rates were measured in both DANs and MBONs. DAN activation rates were obtained during training trials, whereas the MBON activation rates were measured during the test trials (Fig. 4). Further, we compared the model to a recent study in which dendritic MBON activity was measured (Fig. 5; Felsenberg et al., 2018). KC:: MBON synapses in M6 populate the dendritic tree while the MVP2::M6 are located proximal to the dendritic root (Felsenberg et al., 2018). Measuring the calcium activation across the dendritic field can thus be interpreted as the 
overall excitatory $\mathrm{KC}$ input to the MBON. In analogy to the experimental approaches, not only the total synaptic input, but also the sum of excitatory synaptic input from KCs was measured.

\section{Performance at the group level}

Each new initialization of a network involved the random generation of the odor induced PN activation rates (ranging between 0.8 and 1 , evenly distributed) and a random number of $\mathrm{PN}: \mathrm{KC}$ connections (in the range 5-15, evenly distributed) for each $\mathrm{KC}$ to establish variability across individuals.

To test statistical significance of group differences in the performance index, the Wilcoxon rank-sum test was used. To test for differences of MBON activation rates in the same group before and after extinction learning, the Wilcoxon signed-rank test was used. Model results were compared with the experimental results obtained by Felsenberg et al. $(2017,2018)$. To extract single data points from the experimental studies, the GRABIT tool by MathWorks was applied. Model simulation and statistical analyses were performed with MATLAB_R2018b (MathWorks Inc). The full code for the (MB model is available on the GitHub account of the lab: https://github.com/ nawrotlab.

\section{Results}

\section{Circuit model of the Drosophila MB}

We implemented a neural network model of the olfactory memory circuit of $D$. melanogaster (Fig. 1) where individual neurons exhibit trial-resolved activation dynamics (see Materials and Methods). Our network model integrates experimentally confirmed connections in the adult fruit fly. It involves three feed-forward layers of the antennal lobe PNs, the MB KCs, and four individual MBONs with specific lateral inhibitory connections. Reinforcing stimuli (US) of rewarding or punishing nature are mediated by two DANs. Each DAN receives specific feedback input from a single excitatory MBON and exerts a recurrent neuromodulatory effect on the plastic KC::MBON synapses.

Stimulation with a particular odor is modeled as one specific input pattern activating 50 out of total 100 PNs, each with a random activation rate (see Materials and Methods). Similarity between different odors was defined as the percentage of overlap between the PN activation patterns (Fig. 2C). This establishes a dense combinatorial odor code in the PN layer as reported experimentally for fruit flies (Wilson et al., 2004; Olsen et al., 2010) and other species, notably the honeybee (Joerges et al., 1997; Krofczik et al., 2009), the locust (Mazor and Laurent, 2005; Broome et al., 2006), and the moth (Namiki and Kanzaki, 2008).

The connectivity between the PNs and KCs is divergent-convergent and random (Caron et al., 2013) where each of the $2.000 \mathrm{KCs}$ (Aso et al., 2009) connects to average 10 PNs (uniformly distributed, range of 5-15), matching the experimentally estimated numbers for Drosophila (Leiss et al., 2009; Turner et al., 2008) and establishing a sparse connectivity for each of the postsynaptic KCs. In a second step, a threshold criterion was implemented such that only the $5 \%$ of $\mathrm{KCs}$ with the highest activation rates retain their activation (Fig. $2 \mathrm{C}$ ) while the activation rates of all other KCs was set to zero (Peng and Chittka, 2017). This enforces population sparse coding in the KC layer (Kloppenburg and Nawrot, 2014) as reported in physiological experiments in the fruit fly where, on average, $\sim 5 \%$ of all KCs were activated by a single odor stimulus (Turner et al., 2008; Honegger et al., 2011; Lin et al., 2014).

In the MB output layer, we implemented four out of the 34 anatomically identified MBONs (Ito et al., 1998; Tanaka et al., 2008; Séjourné et al., 2011; Aso et al., 2014a). These are MV2 (MBON- $\beta 1>\alpha / \mathrm{MBON}-06), \mathrm{M} 6$ (MBON- $\left.\gamma 5 \beta^{\prime} 2 \mathrm{a} / \mathrm{MBON}-01\right)$, MVP2 (MBON- $\gamma 1$ pedc $>\alpha / \beta / \mathrm{MBON}-11)$ and V2 (MBON$\alpha^{\prime} 1$, MBON- $\alpha^{\prime} 3$ ap, MBON- $\alpha^{\prime} 3 \mathrm{~m}, \mathrm{MBON}-\alpha 2 \mathrm{sc}$ and MBON$\alpha 2 \mathrm{p} 3 \mathrm{p} / \mathrm{MBON}-15-\mathrm{MBON}-19$ ). The neuron cluster V2, consisting of five neurons, was modeled as a single neuron for simplicity. M6, MV2, MVP2, and V2 have been previously shown to be involved in odor valence coding with $M 6$ and MV2 mediating odor driven avoidance behavior, and MVP2 and V2 promoting approach behavior toward an olfactory stimulus (Séjourné et al., 2011; Aso et al., 2014b; Bouzaiane et al., 2015; Owald et al., 2015; Perisse et al., 2016; Ueoka et al., 2017). All KCs connect to each of the four MBONs (full connectivity; Fig. 1). Our model includes two inhibitory lateral connections among MBONs. The inhibitory synapses between MVP2 and M6 (Perisse et al., 2016; Felsenberg et al., 2018) have been suggested to be functionally relevant for aversive memory extinction (Felsenberg et al., 2018). We additionally assume a symmetric lateral inhibitory connection from MV2 to the V2 neuron. It has been shown that the MV2 neuron projects onto $\mathrm{MBON}-\alpha 2 \mathrm{sc}$ and $\mathrm{MBON}-\alpha 2 \mathrm{p} 3 \mathrm{p}$, which are part of the V2 cluster and it was hypothesized that the glutamatergic MV2 acts inhibitory on both neurons (Aso et al., 2014a,b), as has been shown for glutamatergic neurons in the AL (Liu and Wilson, 2013).

\section{Reinforcing pathways and synaptic plasticity}

In our circuit model, the presence of the appetitive or the aversive US are signaled by two distinct neuromodulatory DANs, the PPL1 and PAM neuron, respectively (Fig. 1). The single PPL1 neuron is representative of the PPL1 neuron cluster activated by aversive sensory stimuli such as electric shock (Claridge-Chang et al., 2009; Mao and Davis, 2009; Aso et al., 2010, 2012), the PAM neuron represents the PAM cluster that is activated by appetitive sensory stimuli such as sucrose (Burke et al., 2012; Liu et al., 2012; Waddell, 2013; Yamagata et al., 2015). Both neurons receive additional excitatory input from either the M6 or the V2 MBON (Ichinose et al., 2015; Eichler et al., 2017; Felsenberg et al., 2017, 2018; Eschbach et al., 2020).

The presence of a reinforcing signal (US) has differential effects on the DAN activation. A rewarding US leads to an excitation of PAM and can reduce excitation of PPL1 input (Eq. 5a). Vice versa, a punishing reinforcer excites PPL1 and at the same time can modulate excitation of PAM input (Eq. 5b). This modulatory effect in our model is 
based on an experimental study revealing that a negative reinforcement inhibits $\mathrm{PAM}-\gamma 4$ and $\mathrm{PAM}-\gamma 5$ neurons, whereas sugar feeding inhibits PPL1- $\gamma 2$ and PAM- $\gamma 3$ (Cohn et al., 2015).

Plasticity in our model exclusively resides in the $\mathrm{KC}:$ : MBON synapses, reflecting the prevalent data-based hypothesis (see Discussion). These synapses are subject to plasticity according to the learning rule in Equations $7 \mathrm{a}$, $7 \mathrm{~b}$. In the initial state of the model, the weights of all KC:: MBON synapses are set to the same fixed value. In a given experimental trial, and for any synapse KC::MV2 or $\mathrm{KC}: \mathrm{M6}$, plasticity occurs when activation of the presynaptic $\mathrm{KC}$ coincides with activation of the reward-mediating PAM neuron. Likewise, any KC::V2 or KC::MVP2 synapse undergoes changes when the presynaptic KC and the punishment-mediating PPL1 neuron are active at the same time. The reduction of a synaptic weight is proportional to the DAN activation rate (for details, see Materials and Methods). This establishes two distinct parallel but interconnected neuromodulatory pathways, each involving feedback from MBONs to the DANs that, in turn, can modulate $\mathrm{KC}: \mathrm{MBON}$ synapses (Fig. 1).

\section{Appetitive and aversive conditioning establish a behavioral odor preference}

In a first set of experiments, we subjected our model to a classical conditioning protocol. This protocol (Fig. 2A) mimics standard training procedures in the fruit fly (see Materials and Methods). In each trial the trained odor, $\mathrm{CS}+$, was paired with either reward or punishment (US). Subsequently a second odor stimulus was presented without reinforcement (CS-).

After a predefined number of training trials, we performed a memory retention test by presenting the model with the CS+ alone (no reinforcement) and subsequently with the unpaired odor (CS). We computed a preference index (Eq. 8) based on the activation rates of approach and avoidance mediating MBONs for both odors, the CS+ and the CS- (see Materials and Methods). The model performance index (Eq. 9) mimics comparison with the behavioral performance index computed from experiments in the fruit fly.

Olfactory memories are established as a skew in the MBON network: aversive learning reduces $\mathrm{CS}+$ driven responses in approach MBONs (MVP2-MBON and V2 cluster $\mathrm{MBONs}$ ) resulting in a skew toward avoidance. In contrast, appetitive learning skews the network toward approach by reducing CS + mediated input to avoidance coding M4 and M6 MBONs (Aso et al., 2014b; Owald et al., 2015; Owald and Waddell, 2015; Perisse et al., 2016; Zhang et al., 2019).

We explored the preference index during the retention test for a varying number of training trials (Fig. $2 B$ ). In the naive state, i.e., before the first training trial, the model did not reveal a preference. This is because of the balanced synaptic weight initialization of the model. A single appetitive training trial yielded a strong preference toward the rewarded odor, the $\mathrm{CS}+$. With each additional training trial, the preference index further incremented by a small amount and thus additional training led to only a gradual increase of the preference index saturating after $\sim 10-15$ trials. Aversive conditioning revealed a negative preference index reaching a similar absolute level after a single training trial (Fig. 2B, lower panel). Both, single-trial learning and saturation of behavioral performance within few trials matches the experimental observations during associative learning in insects (see Discussion).

For all subsequent experiments, we chose 12 training trials, both for the training and the reactivation phase. This number mimics the standard experimental protocol for aversive conditioning in fruit flies, in which a group of animals is exposed to 12 subsequent electric shocks while constantly being exposed to the CS+ (Tully and Quinn, 1985; Scheunemann et al., 2013; Felsenberg et al., 2018).

\section{Generalization to novel odors}

We next analyzed how the learned preference to the CS+ odor generalizes to novel odors of varying similarity with the CS+ odor by comparing the preference indices toward CS+ and toward a novel odor during the retention test (Fig. 2D). We designed the pattern of activated PNs for each novel odor such that it has a defined overlap with the CS+ pattern, ranging from zero to $80 \%$ overlap (see Materials and Methods). Our results in Figure $2 D$ predict that generalization to a novel odor is rather low even for the highest odor similarity and decays with decreasing odor similarity, effectively reaching zero generalization for odors that share $40 \%$ or less activated PNs with the CS+ odor. For the subsequent extinction learning experiments, we used CS- odors that had $60 \%$ pattern overlap with the CS+ odor.

\section{Extinction learning significantly reduces the conditioned odor response}

We analyzed extinction learning after appetitive and aversive conditioning according to the stimulation proto$\mathrm{col}$ in Figure $3 A$. The initial training phase was followed by a reactivation phase during which the model is repeatedly presented with the CS+ odor alone (no reinforcement). This matches the experimental protocols for appetitive (Felsenberg et al., 2017) and aversive (Felsenberg et al., 2018) conditioning and subsequent extinction learning and allows for a direct comparison of our simulation results with the experimental results, both at the behavioral and the physiological level (compare Extended Data Fig. 3-1).

Repeated reactivation of the CS+ odor alone after either appetitive or aversive conditioning resulted in memory extinction (Fig. 3B,C), i.e., in a significant reduction of the learned CS+ approach or avoidance. In addition, generalization to the CS- odor was abolished (Fig. 3B,C). The observation that learning is induced when the expected reward or punishment is omitted is in line with the idea of prediction error dependent learning (see Discussion). For a quantitative comparison with the behavioral results of the binary memory test in Felsenberg et al. (2017), we computed the performance index of the model by subtracting the preference index for CS- from the preference index for CS+ (see Materials and Methods). The appetitive conditioning protocol in the model and in vivo yielded similar performance indices (model: $0.30 \pm 0.03$ mean \pm 
A

Training

Appetitive Conditioning

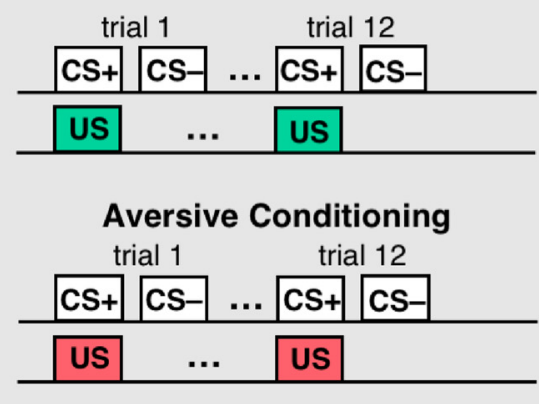

\section{Reactivation}

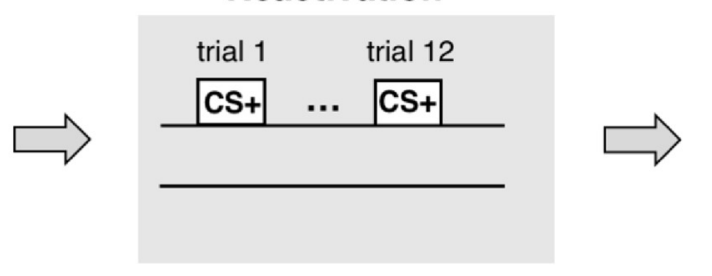

\section{Test}

$\mathrm{CS}+\mathrm{CS}-$

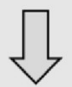

Preference Index \& Performance Index
B

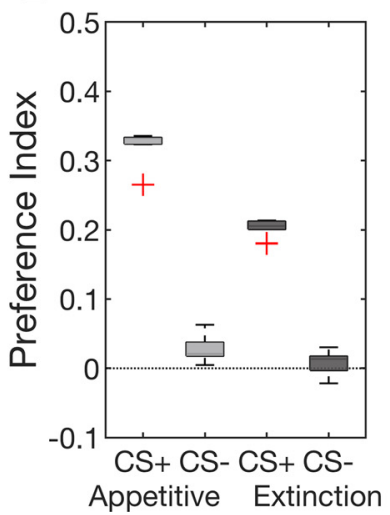

C

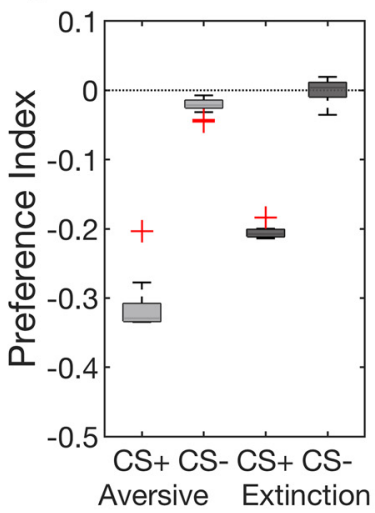

D

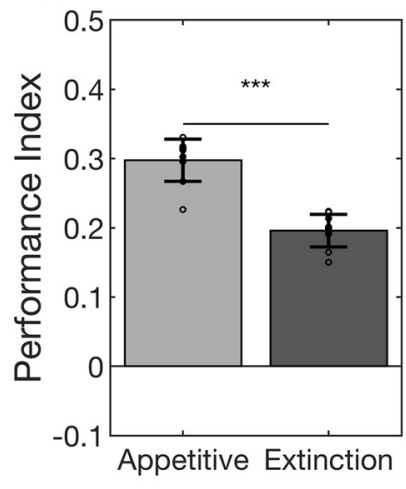

E

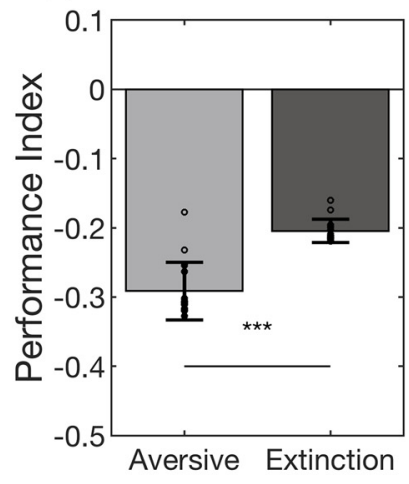

Figure 3. CS+ odor reactivation leads to memory extinction. $\boldsymbol{A}$, Training and test protocol as in Figure $2 A$. In the extinction protocol, the training phase is followed by an odor reactivation phase, in which the CS + was presented alone for 12 consecutive trials before performing the retention test. $\boldsymbol{B}$, The appetitive conditioning protocol (light gray) induced a strong and significant preference for the $\mathrm{CS}+$ odor and a weak and significant preference for the CS- odor. Extinction learning partly abolished the CS + preference during the retention test (dark gray). The preference index for the CS+ odor was significantly lower than after initial appetitive conditioning, for the CS- odor no more significant preference was observed after extinction learning. $\boldsymbol{C}$, Symmetric results were observed for aversive conditioning. Again, extinction learning significantly reduced the learned preference for the CS + odor. Boxplots show the median and the lower and upper quartiles, whiskers indicate 1.5 times interquartile range, outliers are marked with + symbol. $\boldsymbol{D}, \boldsymbol{E}$, The performance indices were significantly reduced after extinction compared with the initial appetitive and aversive memories. Data are presented mean $\pm \mathrm{SD} ;{ }^{* \star *} p<0.001 ; n=15$ independent models. For preference and performance indices of the model tuned toward asymmetric appetitive and aversive learning pathways, see Extended Data Figure 3-2.

SD, experiment: $0.29 \pm 0.05$ mean \pm SEM). Memory extinction led to a significantly reduced approach performance $(0.20 \pm 0.02$; Fig. $2 B)$. However, the effect of memory extinction in the behavioral experiment was considerably stronger, effectively erasing the initial behavioral memory expression (0.01 \pm 0.03 ; Felsenberg et al., 2017). The aversive conditioning protocol led to a negative performance index of $-0.29 \pm 0.04$ (mean $\pm \mathrm{SD}$ ) for the model, which in absolute terms was smaller than the experimental value of $-0.39 \pm 0.04$ (mean \pm SEM; Felsenberg et al., 2018). The reactivation of the CS + odor after the aversive conditioning led to a significantly reduced avoidance behavior $(-0.20 \pm 0.02)$, very similar to the experimental results $(-0.20 \pm 0.03)$. A detailed quantitative comparison of experimental and model results is provided in Extended Data Extended Data Fig. 3-1.

\section{Memory is rapidly established within a single trial}

As a next step, we investigated the dynamics of plasticity during the course of learning and extinction. To this end, we monitored the activation rates of DANs and MBONs and the gross synaptic input to the MBONs across trials during single network simulations for appetitive (Fig. $4 A, B$ ) and aversive (Fig. $4 C, D$ ) conditioning and extinction.

In both, appetitive and aversive conditioning, the first pairing of CS+ and US induced a strong response in either the PAM or PPL1, respectively. This strong neuromodulatory signal resulted in a significant reduction of the respective KC::MBON synapses and led to a strongly reduced KC::MBON input to either MV2/M6 or MVP2/V2 during the second training trial. As a consequence, feedback excitation from M6 to PAM (V2 to PPL1) in appetitive (aversive) learning is abolished from the second trial onward, leading to further synaptic weight modulations that are weak compared with those in the very first training trial. The evolution of plasticity is reflected in the model performance index, indicating a switch-like increase after the first training trial and moderate but steady increase after subsequent trials. Saturation of the learning effect 
becomes visible only after $\sim 10-12$ trials (compare Fig. $2 B$ ). In conclusion, the model learns within a single trial and more training leads to a stronger valence signal as encoded in the imbalance of MV2 and MVP2 activation.

Extinction learning follows a different and slower dynamics in our network model. While already a single presentation of the CS+ odor without reinforcer leads to the induction of the extinction memory and a significant reduction of the performance index (Fig. 4), we observe an only gradual extinction that saturates across the 12 extinction trials used here. No complete extinction is observed in our model.

\section{Associative learning and extinction learning establish two separate memory traces}

We tested the hypothesis that associative learning and subsequent extinction learning form two parallel and distinct memories at the physiological level. To this end, we mimicked experiments in Felsenberg et al. (2018), in which the authors measured $\mathrm{KC}$ input to individual MBONs by means of in vivo calcium-imaging from the dendritic field.

We first consider the case of aversive conditioning and subsequent extinction learning. After initial conditioning, the CS+-induced synaptic input to the MVP2 and V2 neurons is significantly lower than the CS--induced input (Fig. $5 C$ ). The synaptic input to the M6 and MV2 neurons, however, is similar for both odors (Fig. 5D). The extinction protocol, i.e., the subsequent reactivation with CS+ alone (Fig. 3A), did not alter KC synaptic input to MVP2 and V2 (Fig. $5 C$ ). In contrast, CS+-induced synaptic input to M6 and MV2 was significantly reduced after extinction and in comparison to the CS--induced input (Fig. 5D). These results are fully consistent with the in vivo calcium-imaging results by Felsenberg and colleagues (Felsenberg et al., 2018; compare Extended Data Fig. 3-1) who observed a significantly reduced dendritic calcium activation in MVP2 in response to the CS+ stimulus after aversive conditioning, while there was no significant change in $\mathrm{M} 6$ activation (Felsenberg et al., 2018; see their Fig. 3). After memory extinction, the decreased CS+ response in MVP2 remained while additional plasticity was observed as CS+specific reduction in calcium levels in M6.

Our circuit model predicts a symmetric network behavior for the extinction of an appetitive memory. After appetitive conditioning, the CS+-induced excitatory input was lower than the CS--mediated input to the avoidance-mediating M6 and MV2 (Fig. 5B). However, the input to the approach mediating MVP2 and MV2 remained unchanged (Fig. $5 A$ ). Reactivation of the memory by stimulation with the CS + odor alone led to a reduction of the excitatory dendritic input into MVP2 and V2 (Fig. $5 A$ ). As a result, the $\mathrm{CS}+$ preference and performance indices acquired during initial conditioning were significantly reduced after memory extinction (Fig. 3B,D).

\section{Distinct pathways are crucial for appetitive and aversive memory extinction}

To validate the idea of recurrent feedback as a teaching signal in our model we tested which neurons are necessary for extinction learning. To this end, we mimicked shibire ${ }^{\text {ts1 }}$ experiments performed by Felsenberg et al. $(2017,2018)$, who selectively suppressed the activity of individual neurons or neuron clusters during CS+ odor reactivation (see Materials and Methods; Fig. 6).

We first tested extinction of appetitive memory. Selective blocking of PPL1-cluster or V2-cluster during the reactivation phase abolished the extinction of an appetitive memory. In contrast, blocking of PAM had no effect on extinction (Fig. 6A). These findings agree with the experimental results (Extended Data Fig. 3-1) in Felsenberg et al. (2017). Conversely, in the extinction of aversive memory, Felsenberg et al. (2018) showed that the behavioral effect of extinguishing the aversive memory was significantly diminished by either blocking the PAM cluster or the M6 neurons. However, blocking the vesicle release machinery of the PPL1 cluster had no effect on aversive memory extinction (Extended Data Fig. 3-1). Our model showed the same qualitative result where blocking of either the M6 or the PAM but not of the PPL1 neuron abolished the extinction of an aversive memory (Fig. 6B).

To formulate novel predictions for future experiments we further analyzed our model by silencing other MBONs during the reactivation phase (Fig. 6; Extended Data Fig. 3-1). Deactivating V2 during the reactivation phase did not significantly alter the aversive extinction memory. Conversely, a blockage of M6 during the extinction process had no effect on the appetitive extinction memory performance either. Blocking MVP2 or MV2 during odor reactivation had no effect on both, appetitive and aversive memory extinction. Thus, our model predicts that a single MBON, V2, or M6, is exclusively involved in the formation of the appetitive or aversive extinction memory, respectively.

\section{$\mathrm{KC}$ activity is partly required for extinction learning}

We additionally investigated the role of KCs in extinction learning in our model. To this end, we applied the shibire ${ }^{\text {ts1 }}$ protocol to $\mathrm{KCs}$. When we blocked all KCs during the training phase, neither appetitive nor extinction memory could be established. However, when we randomly chose and blocked $50 \%$ of all KCs during appetitive and aversive extinction training, we did not observe an effect on extinction memory acquisition (Fig. 6). Thus, partially blocking $\mathrm{KC}$ activity during odor reactivation might not or only mildly interfere with extinction learning while an almost complete block of all KCs should prevent extinction learning in the fly (Extended Data Fig. 6-1; see also Discussion).

\section{Discussion}

In the present study, we established a circuit model of the fruit fly based on confirmed anatomy and physiology. It can explain formation and extinction of an olfactory memory, and single-trial learning. We discuss the implications, limitations and predictions of our model.

\section{Prediction error coding}

The prediction error theory hypothesizes that learning takes place if there is an unexpected change in the 
A

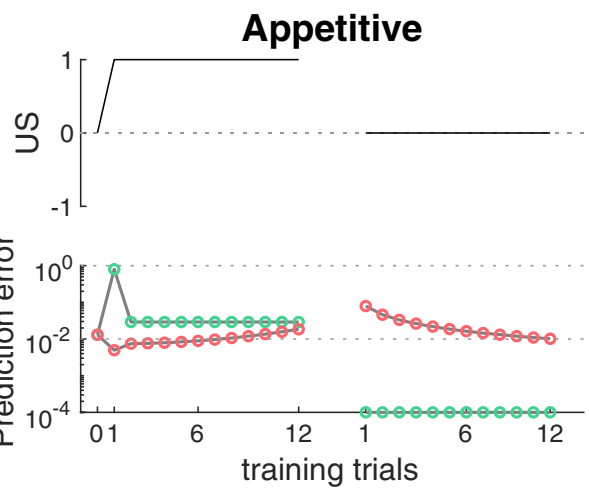

B
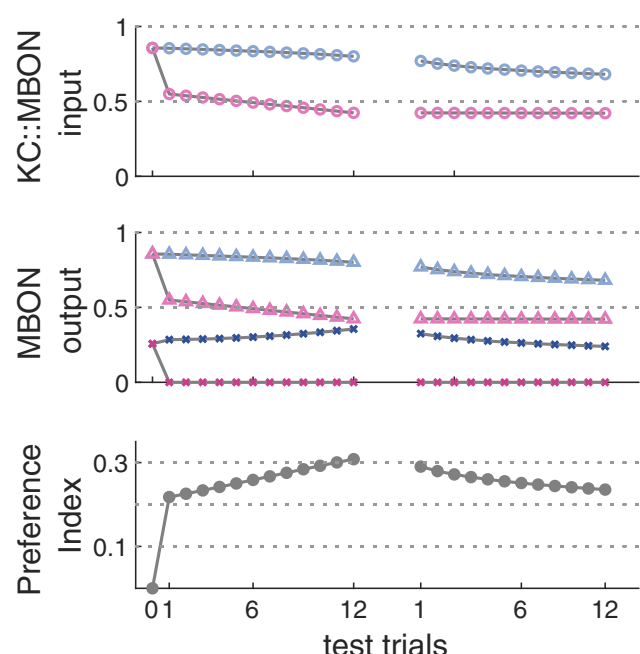

C
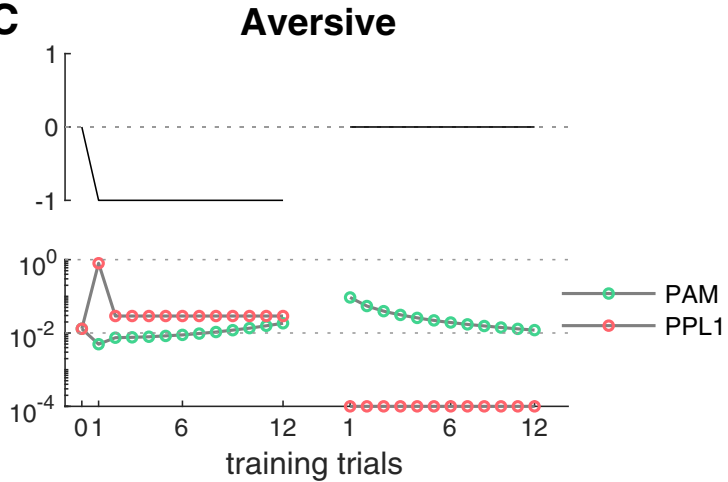

D
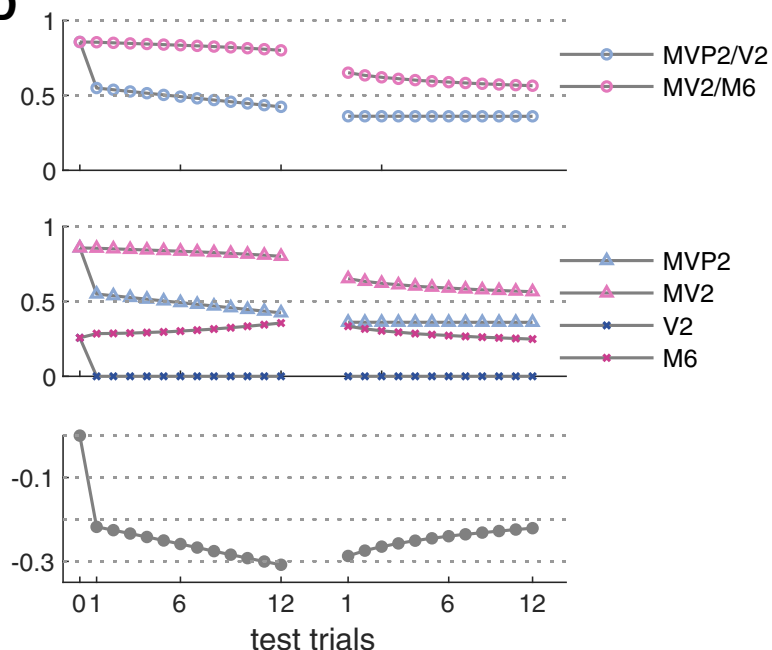

Figure 4. Neuron activation rates and behavioral performance across trials. $\boldsymbol{A}$, Reward value (US, top) and DAN activation rates (bottom) across 12 initial conditioning trials (left) and 12 extinction trials (right). Trial zero indicates neuron activation rates in the naive model before learning. Reward presentation resulted in a prominent peak activation of the PAM during the very first training trial, i.e., when the actual reward strongly deviates from the predicted reward. Omission of the reward during the first extinction trial (right) resulted in a step-like reduction of PAM activation the simultaneous increase in PPL1 activation, which is crucial for extinction learning. $\boldsymbol{B}$, Summed synaptic KC input to MBONs (top), MBON activation rates (middle), and model preference index (bottom) for each simulated test trial that followed the respective training trial (see Materials and Methods). The strong PAM activation during the first conditioning trial resulted in a strong change of the synaptic weights between KCs and MV2 and M6, and consequently in a step-like reduction of the synaptic input and the output activation rates. Consequently, the model preference index represents the imbalance between M6 and MV2 activation rates showed a step-like increase predicting a switch-like expression of a CR behavior after only a single conditioning trial. Additional training led to a further gradual reduction of gross synaptic input to and activation of MV2 and M6, paralleled by the gradual increase of the preference index. Extinction learning led to a gradual reduction of synaptic weights in the KC::MVP2 and KC::V2 pathway. This reduces the difference between M6 and MV2 activation and leads to a gradual extinction of the preference index. $\boldsymbol{C}, \boldsymbol{D}$, Same as $\boldsymbol{A}, \boldsymbol{B}$ for aversive conditioning (left) and subsequent extinction (right). Simulation results are shown for a single network. This was initiated identically before appetitive $(\boldsymbol{A}, \boldsymbol{B})$ and aversive $(\boldsymbol{C}, \boldsymbol{D})$ conditioning to enforce identical initial conditions stressing the symmetric mechanism of reward prediction.

valence of a stimulus (Rescorla and Wagner, 1972). The model performance index in Figure 4 computed after each training trial mimics behavioral performance across trials and fits the prediction error theory and experimental studies. The behavioral learning effect is reduced across trials and the learning curve saturates with increased training. The results match the well-known saturation in the conditioned response (CR) behavior in the honeybee (Pamir et al., 2011). This effect, typically observed across a group of animals, has been formalized in the RescorlaWagner model (Rescorla and Wagner, 1972). A recent rate-based model of the fly MB (Bennett et al., 2021) assumed that connections from MBONs to DANs are crucial for asymptotic learning based on reward prediction. Our model proposes that prediction error coding in $D$. melanogaster is achieved through a network mechanism supported by the specific circuit motif sketched in Figure 1. It is suggested that DANs receive relevant information not only from sensory neurons directly but also via odor activated MBONs (Felsenberg et al., 2017, 2018). It was implemented in the model through excitatory connections from laterally connected MBONs to DANs. Initial conditioning of the model leads to activation of the PAM (PPL1) DAN and therefore induces downregulation of the aversive (appetitive) MBONs. This learning effect declines after the first learning trial, since MBON 

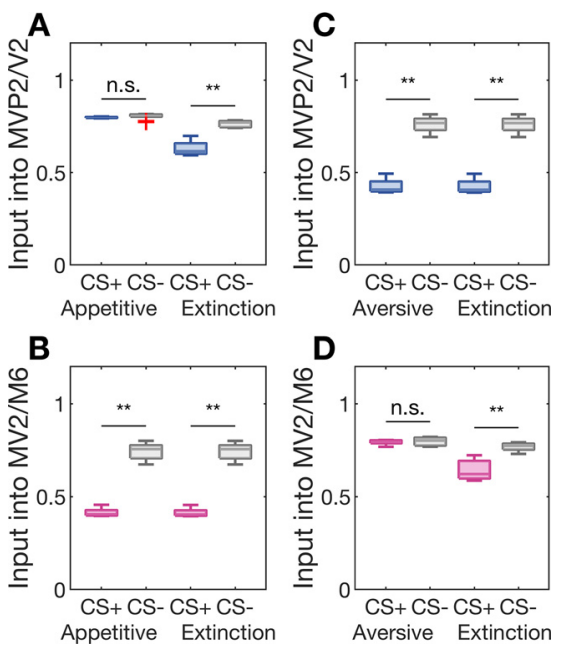

Figure 5. Two separate memories underlie initial and extinction learning. CS+ odor-induced activation rates of MBONs were measured before and after extinction training to locate associative and extinction memories. $\boldsymbol{A}, \boldsymbol{B}$, Appetitive learning led to a relative depression of $\mathrm{CS}+$ odor-induced activation rates of MV2 and M6, but not MVP2 and V2 MBONs. The memory trace in MV2/M6 stayed intact even after appetitive memory was extinguished. Extinction decreased the CS+ response of MVP2/ V2, establishing a parallel memory trace. $\boldsymbol{C}, \boldsymbol{D}$, Aversive conditioning led to a reduced CS + input into MVP2/V2, but not into MV2/M6. After memory extinction, the memory trace in MVP2/ V2 remained, and we observed an additional decrease in CS + response in MV2/M6. Boxplots show the median and the lower and upper quartiles, whiskers indicate 1.5 times interquartile range, outliers are marked with + symbol; n.s. $=$ not significant, ${ }^{* *} p<$ 0.01 . Results across simulation of $n=10$ networks in all panels.

activity is not sufficient to activate DANs after being downregulated.

\section{Single-trial learning}

Learning within a single trial is a fundamental ability of vertebrates (Irwin et al., 1968; Cook and Fagot, 2009) and invertebrates, and the underlying mechanisms are at least partially conserved across phyla. In insects, single-trial learning has been intensely studied in classical and operant olfactory, tactile, and visual conditioning of the honeybee Apis mellifera (Menzel et al., 1974; Smith, 1991; Sandoz et al., 1995; Menzel, 1999; Pamir et al., 2014; Villar et al., 2020). Appetitive olfactory conditioning of the proboscis extension response allows the observation of the all-or-none CR behavior during successive training trials where the onset of US presentation is delayed with respect to the onset of the CS odor. Typically, 40-60\% of bees show a CR after a single pairing of odor with reward (Pamir et al., 2014) and a single learning trial is sufficient to establish short-term and long-term memory, which can be recalled up to $3 \mathrm{~d}$ after training (Smith, 1991; Menzel, 1999; Pamir et al., 2014; Villar et al., 2020). Additional training trials lead to a saturation after approximately three to four trials with respect to the fraction of animals that express a CR.

In the fruit fly, single-trial learning has been established more recently. Aversive conditioning of adult flies with electric shock punishment showed that pairing of a single odor presentation (of 10-60 s in duration) with a single electric shock induces short term memory (Beck et al., 2000; Scheunemann et al., 2013) and long-term memory, retrievable for up to $14 \mathrm{~d}$ if the experimental context is kept strictly constant for training and memory test (Zhao et al., 2019). In appetitive conditioning, a single session of odor-reward pairing could establish long-term memory (Krashes and Waddell, 2008; Colomb et al., 2009). Weiglein et al. (2019) could show that also in the fruit fly larva a single session of appetitive conditioning of $5 \mathrm{~min}$ was sufficient to establish a short-term memory where the performance index in the memory test increased with increasing duration (in the order of minutes) of the training session.

In our circuit model, the very first training trial induces a switch-like change in the network response and in the behavioral memory expression. This parallels the switch-like behavioral dynamics observed in learner bees (Pamir et al., 2011) and provides an explanation for the single-trial induction of appetitive and aversive memories in the fruit fly (Krashes and Waddell, 2008; Colomb et al., 2009; Zhao et al., 2019). In contrast, during extinction learning we propose that an extinction memory builds up gradually in the opposing pathway.

\section{Extinction learning}

Experimental studies in the fruit fly have shown that extinction learning establishes distinct and opposing memory traces. The extinction of reward memory requires punishment coding dopamine neurons whereas extinction of aversive memory is mediated by reward coding neurons (Felsenberg et al., 2017, 2018). Recent findings in mice (Salinas-Hernández et al., 2018) and rats (Luo et al., 2018) suggest that this principle for extinction learning is conserved in mammals. The authors showed that DANs from the ventral tegmental area associated with reward signaling are required to extinguish fear memory.

The importance of MBON::DAN feedback to perform complex learning tasks such as extinction has been formalized recently in a rate-based model of the Drosophila larva (Eschbach et al., 2020). In our adult fly model, the MBON::MBON and MBON::DAN connections for the extinction of aversive and appetitive memories are strictly symmetrical. During extinction of an aversive memory the dopaminergic reward signal of the PAM neuron in extinction trials is driven by excitation from the M6 MBON, which receives reduced inhibition from the approach mediating MVP2 as a result of the initial aversive conditioning that reduced KC drive of MVP2. Thus, extinction learning establishes a reward-like extinction memory trace in parallel to the initial aversive memory trace. Intriguingly, the initial memory trace is not altered by extinction (Fig. 5). This matches the experimental results and the proposed mechanism in Felsenberg et al. (2018) supporting the long-standing hypothesis of two parallel memory traces after extinction (Bouton, 2004; Dudai, 2004; Eisenhardt and Menzel, 2007).

The strict symmetry of the recurrent pathways in our model has the consequence of symmetrical quantitative results for the performance index and neuron input activation rates (Extended Data Fig. 3-1). The experimental 
A

Appetitive Conditioning
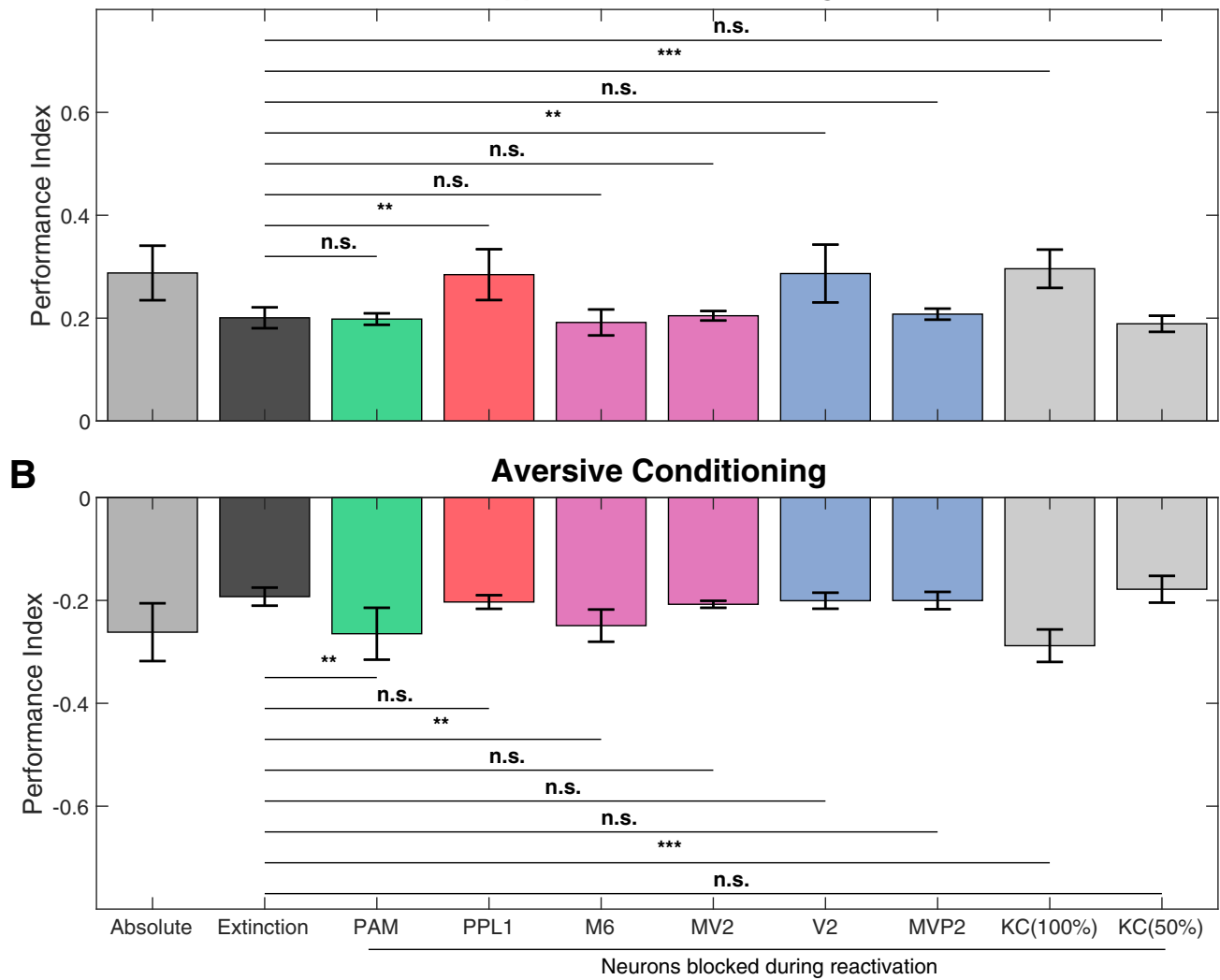

Figure 6. Blocking specific neuron groups can reverse memory extinction. The computational model was used to reproduce the neurogenetic manipulations with temperature-sensitive shibire ${ }^{\text {ts } 1}$ mutant flies. The activation of individual neurons was suppressed during extinction training only. This allows to identify neurons that are crucial to the process of establishing the extinction memory in our model. $\boldsymbol{A}$, Knocking down PPL1 or V2 during extinction training led to a completely abolished the appetitive extinction memory. Blocking PAM, M6, MV2, or MVP2 neurons during odor re-exposure had no effect on the appetitive extinction memory. Blockage of all KCs (100\%) prevented the model from extinction learning. However, when only $50 \%$ of the KC population was blocked during odor reactivation, extinction learning remained unaffected. Further effects of partial KC blocking on extinction learning are presented in Extended Data Figure 6-1. B, Suppressing the activity of PAM DANs, M6 neurons, or KCs (100\%) during CS+ odor re-exposure after aversive conditioning prevents the model from forming the aversive extinction memory. However, blocking PPL1 DANs, MV2, V2, MVP2, or KCs (50\%) does not diminish the memory extinction. Data are presented as mean \pm SD; n.s. = not significant, ${ }^{* \star} p<0.01,{ }^{* * *} p<0.001 ; n=15$.

situation, however, does not provide fully symmetric behavioral results. Our model matches well in the aversive memory pathway, i.e., for appetitive conditioning and extinction of aversive memory. In aversive conditioning experiments, however, Felsenberg et al. (2017, 2018) obtained stronger performance indices than our model and extinction of the appetitive memory was complete (Extended Data Fig. 3-1). Independent tuning of model parameters for the two parallel pathways in our symmetric model allows for an improved quantitative match with the experimental results (Extended Data Fig. 3-2). However, a complete fit to the experimental data were not achievable. In particular, the complete extinction of the appetitive memory cannot be obtained. We thus conclude that the current model lacks at least on additional mechanism that introduces asymmetry in circuit and behavior. Our minimal model does not include all MBONs and DANs and their proven interconnections (Takemura et al., 2017), i.e., the inhibitory connection between MVP2 and the PPL1 cluster, might add functional asymmetry between the appetitive and the aversive memory pathway (Perisse et al., 2016; Pavlowsky et al., 2018).

\section{Limitations of the model}

Our network model (Fig. 1) can be viewed as a minimal circuit model of the MB sufficient to explain prediction error coding, single-trial conditioning and extinction learning. We focused on only four out of $34 \mathrm{MBONs}$ and restricted MB connectivity to feedforward from KCs to four MBONs and feedback from MBONs to DANs but did not explore other synaptic contacts among $\mathrm{KCs}$ and between $\mathrm{KCs}, \mathrm{MBONs}$ and DANs within the MB lobes as uncovered in the fly EM connectome (Takemura et al., 2017). Learning in our model is achieved through reinforcementmediated plasticity at a single synaptic site, which has been shown to be involved in appetitive and aversive short-term olfactory memory (Zars et al., 2000; Pascual 
and Préat, 2001; Owald and Waddell, 2015). Increasing experimental evidence indicates that olfactory learning can involve plasticity at multiple sites within the insect MB (Pascual and Préat, 2001; Kremer et al., 2010; Trannoy et al., 2011; Bouzaiane et al., 2015; Haenicke et al., 2018; Yamazaki et al., 2018). Also, it has been shown that memories can co-exist on different time scales representing short-term, mid-term, and long-term memories (Pascual and Préat, 2001; Davis, 2011; Trannoy et al., 2011; Yamagata et al., 2015). Our trial-based model approach does not accommodate explicit time scales that would allow to differentiate between simultaneous, delay and trace conditioning (Dylla et al., 2013), memory acquisition and consolidation (Felsenberg et al., 2017) or decay (Shuai et al., 2015). Future extensions may retain full temporal dynamics, e.g., by using spiking neural network models that have previously been used successfully to study classical conditioning in fruit flies (Smith et al., 2008; Wessnitzer et al., 2012; Faghihi et al., 2017; Gupta et al., 2018; Rapp and Nawrot, 2020).

In the present study, we aimed at reproducing the behavioral performance index that is measured in a group forced choice paradigm (Quinn et al., 1974) and thus represents a population measure across individuals, each performing either the correct or the incorrect behavioral choice. The long-held notion states that expression of the $\mathrm{CR}$ behavior in individual flies is stochastic and follows the group-averaged behavior (Quinn et al., 1974) has been questioned by Chabaud et al. (2010) and by studies in the honeybee (Pamir et al., 2011, 2014; Haenicke et al., 2018) and in the cockroach (Arican et al., 2020). They have shown that the average $\mathrm{CR}$ in a group of animals does not accurately reflect memory expression in individuals. Rather, it confounds two subgroups of learners and non-learners where learners showed a switch-like and stable expression of the CR behavior mostly after a single trial (average 1.7 trials) with high memory retention rates (>90\%). Different parameters may account for individual learning abilities such as internal state, e.g., of hunger and satiety (Pamir et al., 2014; Sayin et al., 2018) that could influence perception or motivation. Establishing neural circuit models that take into account individuality of learning and individual parameters that influence memory formation is a challenge we aim to address in future model studies.

\section{Model predictions}

Our model makes several predictions that can be tested experimentally. First, we hypothesize that DANs in the PAM and PPL1 cluster compute a prediction error. Specifically, in naive animals and for the example of appetitive learning the PAM should show spontaneous baseline spiking activity and no stimulus response during the presentation of a novel odor. During a first pairing of the CS+ odor with a reward (US) the PAM cluster should show a clear odor response, which will be strongly reduced after the first learning trials (Fig. $4 A$ ). When reward is omitted during extinction trials or in the memory test, we predict a response in which the PAM reduces its spiking output below spontaneous level. This prediction matches the observation in DANs in the monkey
(Schultz et al., 1997) and in the octopaminergic VUMmx1 neuron in the honeybee (Montague et al., 1995; Hammer, 1997), although the circuit mechanisms hypothesized here differs from that proposed by Schultz et al. (1997) and Hammer (1997).

Second, we hypothesize a switch-like induction of a memory trace during a single training trial, implying a steplike change in the MBON activation rates (Fig. 4). A second or third training trial should only induce gradual changes in cellular physiology. Likewise, the behavioral memory expression should be observable after a single learning trial.

Third, our systematic analysis of blocking experiments (Fig. 6) for all MBONs indicate that MV2, M6, and MVP2 do not play a role during appetitive memory extinction, whereas MVP2, MV2, and V2 are dispensable for aversive extinction learning. This could be experimentally tested by blocking these MBONs specifically during extinction trials only. While the co-existence of two memory traces after extinction of an aversive memory has been shown in calcium-imaging from MVP2 and M6 (Felsenberg et al., 2018; Fig. 5D) our model predicts parallel memory traces also for extinction of an appetitive memory (Fig. 5A,B).

Finally, in a study by Schwaerzel et al. (2002), in which $\mathrm{KCs}$ were blocked during aversive conditioning and odor reactivation, the authors argued that $\mathrm{KC}$ activity is dispensable for the acquisition of an extinction memory. However, the two GAL4 lines used in this study include $<50 \%$ of all KCs ( 700 and 850 cells). Our model analysis predicts that a sizable fraction of the $\mathrm{KC}$ population is required and sufficient to form an extinction memory (Fig. 6; Extended Data Fig. 6-1). A complete block of KCs however would interrupt the PN::KC::MBON::DAN signaling pathway and extinction memory could not be acquired. This could tested in a future blocking experiment using, e.g., the OK107-GAL4 line that has been reported to label practically all KCs (Aso et al., 2009).

\section{References}

Arican C, Bulk J, Deisig N, Nawrot MP (2020) Cockroaches Show Individuality in Learning and Memory During Classical and Operant Conditioning. Front Physiol 10:825265.

Aso Y, Grübel K, Busch S, Friedrich AB, Siwanowicz I, Tanimoto H (2009) The mushroom body of adult Drosophila characterized by GAL4 drivers. J Neurogenet 23:156-172.

Aso Y, Siwanowicz I, Bräcker L, Ito K, Kitamoto T, Tanimoto H (2010) Specific dopaminergic neurons for the formation of labile aversive memory. Curr Biol 20:1445-1451.

Aso Y, Herb A, Ogueta M, Siwanowicz I, Templier T, Friedrich AB, Ito K, Scholz H, Tanimoto H (2012) Three Dopamine pathways induce aversive odor memories with different stability. PLoS Genet 8: e1002768.

Aso Y, Hattori D, Yu Y, Johnston RM, lyer NA, Ngo TTB, Dionne H, Abbott LF, Axel R, Tanimoto H, Rubin GM (2014a) The neuronal architecture of the mushroom body provides a logic for associative learning. Elife 3:e04577.

Aso Y, Sitaraman D, Ichinose T, Kaun KR, Vogt K, Belliart-Guérin G, Plaçais PY, Robie AA, Yamagata N, Schnaitmann C, Rowell WJ, Johnston RM, Ngo TT, Chen N, Korff W, Nitabach MN, Heberlein $\mathrm{U}$, Preat T, Branson KM, Tanimoto H, et al. (2014b) Mushroom body output neurons encode valence and guide memory-based action selection in Drosophila. Elife 3:e04580. 
Beck CDO, Schroeder B, Davis RL (2000) Learning performance of normal and mutant Drosophila after repeated conditioning trials with discrete stimuli. J Neurosci 20:2944-2953.

Bennett JEM, Philippides A, Nowotny T (2021) Learning with reward prediction errors in a model of the Drosophila mushroom body. Nat Commun 12:2569.

Bitterman ME, Menzel R, Fietz A, Schafer S (1983) Classical conditioning of proboscis extension in honeybees (Apis mellifera). $\mathrm{J}$ Comp Psychol 97:107-119.

Bouton ME (2004) Context and behavioral processes in extinction. Learn Mem 11:485-494.

Bouton ME (2017) Extinction: behavioral mechanisms and their implications. In: Learning and memory: a comprehensive reference, Ed 2. San Diego: Academic Press.

Bouzaiane E, Trannoy S, Scheunemann L, Plaçais PY, Preat T (2015) Two independent mushroom body output circuits retrieve the six discrete components of Drosophila aversive memory. Cell Rep 11:1280-1292.

Brill MF, Rosenbaum T, Reus I, Kleineidam CJ, Nawrot MP, Rössler W (2013) Parallel processing via a dual olfactory pathway in the honeybee. J Neurosci 33:2443-2456.

Broome BM, Jayaraman V, Laurent G (2006) Encoding and decoding of overlapping odor sequences. Neuron 51:467-482.

Burke CJ, Huetteroth W, Owald D, Perisse E, Krashes MJ, Das G, Gohl D, Silies M, Certel S, Waddell S (2012) Layered reward signalling through octopamine and dopamine in Drosophila. Nature 492:433-437.

Caron SJC, Ruta V, Abbott LF, Axel R (2013) Random convergence of olfactory inputs in the Drosophila mushroom body. Nature 497:113-117.

Chabaud MA, Preat T, Kaiser L (2010) Behavioral characterization of individual olfactory memory retrieval in Drosophila melanogaster. Front Behav Neurosci 4:192.

Chiamulera C, Hinnenthal I, Auber A, Cibin M (2014) Reconsolidation of maladaptive memories as a therapeutic target: pre-clinical data and clinical approaches. Front Psychiatry 5:107.

Claridge-Chang A, Roorda RD, Vrontou E, Sjulson L, Li H, Hirsh J, Miesenböck G (2009) Writing memories with light-addressable reinforcement circuitry. Cell 139:405-415.

Cohn R, Morantte I, Ruta V (2015) Coordinated and compartmentalized neuromodulation shapes sensory processing in Drosophila. Cell 163:1742-1755.

Colomb J, Kaiser L, Chabaud MA, Preat T (2009) Parametric and genetic analysis of Drosophila appetitive long-term memory and sugar motivation. Genes Brain Behav 8:407-415.

Cook R, Fagot J (2009) First trial rewards promote 1-trial learning and prolonged memory in pigeon and baboon. Proc Natl Acad Sci USA 106:9530-9533.

Davis RL (2011) Traces of Drosophila memory. Neuron 70:8-19.

Delamater AR, Westbrook RF (2014) Psychological and neural mechanisms of experimental extinction: a selective review. Neurobiol Learn Mem 108:38-51.

Dudai Y (2004) The neurobiology of consolidations, or, how stable is the engram? Annu Rev Psychol 55:51-86.

Dylla KV, Galili DS, Szyszka P, Lüdke A (2013) Trace conditioning in insects-keep the trace! Front Physiol 4:67.

Eichler K, Li F, Litwin-Kumar A, Park Y, Andrade I, Schneider-Mizell CM, Saumweber T, Huser A, Eschbach C, Gerber B, Fetter RD, Truman JW, Priebe CE, Abbott LF, Thum AS, Zlatic M, Cardona A (2017) The complete connectome of a learning and memory centre in an insect brain. Nature 548:175-182.

Eisenhardt D (2014) Molecular mechanisms underlying formation of long-term reward memories and extinction memories in the honeybee (Apis mellifera). Learn Mem 21:534-542.

Eisenhardt D, Menzel R (2007) Extinction learning, reconsolidation and the internal reinforcement hypothesis. Neurobiol Learn Mem 87:167-173

Eschbach C, Fushiki A, Winding M, Schneider-Mizell CM, Shao M, Arruda R, Eichler K, Valdes-Aleman J, Ohyama T, Thum AS, Gerber B, Fetter RD, Truman JW, Litwin-Kumar A, Cardona A,
Zlatic M (2020) Recurrent architecture for adaptive regulation of learning in the insect brain. Nat Neurosci 23:544-555.

Faghihi F, Moustafa AA, Heinrich R, Wörgötter F (2017) A computational model of conditioning inspired by Drosophila olfactory system. Neural Netw 87:96-108.

Felsenberg J, Barnstedt O, Cognigni P, Lin S, Waddell S (2017) Reevaluation of learned information in Drosophila. Nature 544:240244.

Felsenberg J, Jacob PF, Walker T, Barnstedt O, Edmondson-Stait AJ, Pleijzier MW, Otto N, Schlegel P, Sharifi N, Perisse E, Smith CS, Lauritzen JS, Costa M, Jefferis GSXE, Bock DD, Waddell S (2018) Integration of parallel opposing memories underlies memory extinction. Cell 175:709-722.e15.

Gupta A, Faghihi F, Moustafa AA (2018) Computational models of olfaction in fruit flies. In: Computational models of brain and behavior (Moustafa AA, ed), Ed 1, Vol 1, pp 199-213. Hoboken: Wiley.

Haenicke J, Yamagata N, Zwaka H, Nawrot MP, Menzel R (2018) Neural correlates of odor learning in the presynaptic microglomerular circuitry in the honeybee mushroom body. eNeuro 5: ENEURO.0128-18.2018-13.

Hammer M (1997) The neural basis of associative reward learning in honeybees. Trends Neurosci 20:245-252.

Honegger KS, Campbell RAA, Turner GC (2011) Cellular-resolution population imaging reveals robust sparse coding in the Drosophila mushroom body. J Neurosci 31:11772-11785.

Ichinose T, Aso Y, Yamagata N, Abe A, Rubin GM, Tanimoto H (2015) Reward signal in a recurrent circuit drives appetitive longterm memory formation. Elife 4:e10719.

Irwin S, Banuazizi A, Kalsner S, Curtis A (1968) One trial learning in the mouse. Psychopharmacologia 12:286-302.

Ito K, Suzuki K, Estes P, Ramaswami M, Yamamoto D, Strausfeld NJ (1998) The organization of extrinsic neurons and their implications in the functional roles of the mushroom bodies in Drosophila melanogaster Meigen. Learn Mem 5:52-77.

Joerges J, Küttner A, Galizia CG, Menzel R (1997) Representations of odours and odour mixtures visualized in the honeybee brain. Nature 387:285-288.

Kloppenburg P, Nawrot MP (2014) Neural coding: sparse but on time. Curr Biol 24:R957-R959.

Krashes MJ, Waddell S (2008) Rapid consolidation to a radish and protein synthesis-dependent long-term memory after single-session appetitive olfactory conditioning in Drosophila. J Neurosci 28:3103-3113

Kremer MC, Christiansen F, Leiss F, Paehler M, Knapek S, Andlauer TFM, Förstner F, Kloppenburg P, Sigrist SJ, Tavosanis G (2010) Structural long-term changes at mushroom body input synapses. Curr Biol 20:1938-1944.

Krofczik S, Menzel R, Nawrot MP (2009) Rapid odor processing in the honeybee antennal lobe network. Front Comput Neurosci 2:9.

Leiss F, Groh C, Butcher NJ, Meinertzhagen IA, Tavosanis G (2009) Synaptic organization in the adult Drosophila mushroom body calyx. J Comp Neurol 517:808-824.

Lin AC, Bygrave A, de Calignon A, Lee T, Miesenböck G (2014) Sparse, decorrelated odor coding in the mushroom body enhances learned odor discrimination. Nat Neurosci 17:559-568.

Liu C, Plaçais PY, Yamagata N, Pfeiffer BD, Aso Y, Friedrich AB, Siwanowicz I, Rubin GM, Preat T, Tanimoto H (2012) A subset of dopamine neurons signals reward for odour memory in Drosophila. Nature 488:512-516.

Liu WW, Wilson RI (2013) Glutamate is an inhibitory neurotransmitter in the Drosophila olfactory system. Proc Natl Acad Sci USA 110:10294-10299.

Luo R, Uematsu A, Weitemier A, Aquili L, Koivumaa J, McHugh TJ, Johansen JP (2018) A dopaminergic switch for fear to safety transitions. Nat Commun 9:1-11.

Mao Z, Davis RL (2009) Eight different types of dopaminergic neurons innervate the Drosophila mushroom body neuropil: anatomical and physiological heterogeneity. Front Neural Circuits 3:5-17. 
Mazor O, Laurent G (2005) Transient dynamics versus fixed points in odor representations by locust antennal lobe projection neurons. Neuron 48:661-673.

Menzel R (1999) Memory dynamics in the honeybee. J Comp Physiol A Neuroethol Sens Neural Behav Physiol 185:323-340.

Menzel R, Erber J, Masuhr T (1974) Learning and memory in the honeybee. In: Experimental analysis of insect behaviour (Barton Browne L, Ed), pp 195-217. New York: Springer.

Montague PR, Dayan P, Person C, Sejnowski TJ (1995) Bee foraging in uncertain environments using predictive Hebbian learning. Nature 377:725-728.

Myers KM, Davis M (2002) Behavioral and neural analysis of extinction. Neuron 36:567-584.

Namiki S, Kanzaki R (2008) Reconstructing the population activity of olfactory output neurons that innervate identifiable processing units. Front Neural Circuits 2:1-11.

Olsen SR, Bhandawat V, Wilson RI (2010) Divisive normalization in olfactory population codes. Neuron 66:287-299.

Owald D, Waddell S (2015) Olfactory learning skews mushroom body output pathways to steer behavioral choice in Drosophila. Curr Opin Neurobiol 35:178-184.

Owald D, Felsenberg J, Talbot CB, Das G, Perisse E, Huetteroth W, Waddell S (2015) Activity of defined mushroom body output neurons underlies learned olfactory behavior in Drosophila. Neuron 86:417-427.

Pamir E, Chakroborty NK, Stollhoff N, Gehring KB, Antemann V, Morgenstern L, Felsenberg J, Eisenhardt D, Menzel R, Nawrot MP (2011) Average group behavior does not represent individual behavior in classical conditioning of the honeybee. Learn Mem 18:733-741.

Pamir E, Szyszka P, Scheiner R, Nawrot MP (2014) Rapid learning dynamics in individual honeybees during classical conditioning. Front Behav Neurosci 8:313.

Pascual A, Préat T (2001) Localization of long-term memory within the Drosophila mushroom body. Science 294:1115-1117.

Pavlov IP (1927) Conditioned reflexes: an investigation of the physiological activity of the cerebral cortex. London: Oxford University Press.

Pavlowsky A, Schor J, Plaçais PY, Preat T (2018) A GABAergic feedback shapes dopaminergic input on the Drosophila mushroom body to promote appetitive long-term memory. Curr Biol 28:17831793.e4.

Peng F, Chittka L (2017) A simple computational model of the bee mushroom body can explain seemingly complex forms of olfactory learning and memory. Curr Biol 27:224-230.

Perisse E, Owald D, Barnstedt O, Talbot CBB, Huetteroth W, Waddell S (2016) Aversive learning and appetitive motivation toggle feed-forward inhibition in the Drosophila mushroom body. Neuron 90:1086-1099.

Quinn WG, Harris WA, Benzer S (1974) Conditioned behavior in Drosophila melanogaster. Proc Natl Acad Sci USA 71:708-712.

Quirk GJ, Mueller D (2008) Neural mechanisms of extinction learning and retrieval. Neuropsychopharmacology 33:56-72.

Rapp H, Nawrot MP (2020) A spiking neural program for sensorimotor control during foraging in flying insects. Proc Natl Acad Sci USA 117:28412-28421.

Rescorla RA, Wagner AR (1972) A theory of Pavlovian conditioning: variations in the effectiveness of reinforcement and nonreinforcement. In: Classical conditioning II: current research and theory (Black A and Prokasy WF, eds), pp 64-99. New York: AppletonCentury-Crofts.

Riemensperger T, Völler T, Stock P, Buchner E, Fiala A (2005) Punishment prediction by dopaminergic neurons in Drosophila. Curr Biol 15:1953-1960.

Salinas-Hernández XI, Vogel P, Betz S, Kalisch R, Sigurdsson T, Duvarci S (2018) Dopamine neurons drive fear extinction learning by signaling the omission of expected aversive outcomes. Elife 7: e38818.

Sandoz JC, Roger B, Pham-Delègue MH (1995) Olfactory learning and memory in the honeybee: comparison of different classical conditioning procedures of the proboscis extension response. C R Acad Sci III 318:749-755.

Sayin S, Boehm AC, Kobler JM, de Backer JF, Grunwald Kadow IC (2018) Internal state dependent odor processing and perceptionThe role of neuromodulation in the fly olfactory system. Front Cell Neurosci 12:11-17.

Scheunemann L, Skroblin P, Hundsrucker C, Klussmann E, Efetova M, Schwärzel M (2013) AKAPS act in a two-step mechanism of memory acquisition. J Neurosci 33:17422-17428.

Schmuker M, Pfeil T, Nawrot MP (2014) A neuromorphic network for generic multivariate data classification. Proc Natl Acad Sci USA 111:2081-2086.

Schultz W (2016) Dopamine reward prediction error coding. Dialogues Clin Neurosci 1:23-32.

Schultz W, Dayan P, Montague PR (1997) A neural substrate of prediction and reward. Science 275:1593-1599.

Schwaerzel M, Heisenberg M, Zars T (2002) Extinction antagonizes olfactory memory at the subcellular level. Neuron 35:951-960.

Séjourné J, Plaçais P-Y, Aso Y, Siwanowicz I, Trannoy S, Thoma V, Tedjakumala SR, Rubin GM, Tchénio P, Ito K, Isabel G, Tanimoto $\mathrm{H}$, Preat T (2011) Mushroom body efferent neurons responsible for aversive olfactory memory retrieval in Drosophila. Nat Neurosci 14:903-910.

Shuai Y, Hirokawa A, Ai Y, Zhang M, Li W, Zhong Y (2015) Dissecting neural pathways for forgetting in Drosophila olfactory aversive memory. Proc Natl Acad Sci U S A 112:E6663-E6672.

Smith BH (1991) The olfactory memory of the honeybee Apis mellifera: I. Odorant modulation of short- and intermediate-term memory after single-trial conditioning. J Exp Biol 161:367-382.

Smith D, Wessnitzer J, Webb B (2008) A model of associative learning in the mushroom body. Biol Cybern 99:89-103.

Takemura SY, Aso Y, Hige T, Wong A, Lu Z, Xu CS, Rivlin PK, Hess H, Zhao T, Parag T, Berg S, Huang G, Katz W, Olbris DJ, Plaza S, Umayam L, Aniceto R, Chang LA, Lauchie S, Ogundeyi O, et al. (2017) A connectome of a learning and memory center in the adult Drosophila brain. Elife 6:e26975.

Tanaka NK, Tanimoto H, Ito K (2008) Neuronal assemblies of the Drosophila mushroom body. J Comp Neurol 508:711-755.

Tempel BL, Bonini N, Dawson DR, Quinn WG (1983) Reward learning in normal and mutant Drosophila. Proc Natl Acad Sci USA 80:1482-1486.

Terao K, Mizunami M (2017) Roles of dopamine neurons in mediating the prediction error in aversive learning in insects. Sci Rep 7:1-9.

Trannoy S, Redt-Clouet C, Dura JM, Preat T (2011) Parallel processing of appetitive short- and long-term memories in Drosophila. Curr Biol 21:1647-1653.

Tully T, Quinn WG (1985) Classical conditioning and retention in normal and mutant Drosophila melanogaster. J Comp Physiol A Neuroethol Sens Neural Behav Physiol 157:263-277.

Turner GC, Bazhenov M, Laurent G (2008) Olfactory representations by Drosophila mushroom body neurons. J Neurophysiol 99:734746.

Ueoka Y, Hiroi M, Abe T, Tabata T (2017) Suppression of a single pair of mushroom body output neurons in Drosophila triggers aversive associations. FEBS Open Bio 7:562-576.

Villar ME, Marchal P, Viola H, Giurfa M (2020) Redefining single-trial memories in the honeybee. Cell Rep 30:2603-2613.e3.

Waddell S (2013) Reinforcement signalling in Drosophila; dopamine does it all after all. Curr Opin Neurobiol 23:324-329.

Walsh KH, Das RK, Saladin ME, Kamboj SK (2018) Modulation of naturalistic maladaptive memories using behavioural and pharmacological reconsolidation-interfering strategies: a systematic review and meta-analysis of clinical and 'sub-clinical' studies. Psychopharmacology 235:2507-2527.

Weiglein A, Gerstner F, Mancini N, Schleyer M, Gerber B (2019) Onetrial learning in larval Drosophila. Learn Mem 26:109-120.

Wessnitzer J, Young JM, Armstrong JD, Webb B (2012) A model of non-elemental olfactory learning in Drosophila. J Comput Neurosci 32:197-212. 
Wilson RI (2013) Early olfactory processing in Drosophila: mechanisms and principles. Annu Rev Neurosci 36:217-241.

Wilson RI, Turner GC, Laurent G (2004) Transformation of olfactory representations in the Drosophila antennal lobe. Science 303:366-370.

Yamagata N, Ichinose T, Aso Y, Plaçais PY, Friedrich AB, Sima RJ, Preat T, Rubin GM, Tanimoto H (2015) Distinct dopamine neurons mediate reward signals for short- and long-term memories. Proc Natl Acad Sci USA 112:578-583.

Yamazaki D, Hiroi M, Abe T, Shimizu K, Minami-Ohtsubo M, Maeyama Y, Horiuchi J, Tabata T (2018) Two parallel pathways assign opposing odor valences during Drosophila memory formation. Cell Rep 22:2346-2358.
Zars T, Fischer M, Schulz R, Heisenberg M (2000) Localization of a short-term memory in Drosophila. Science 288:672-675.

Zhang X, Noyes NC, Zeng J, Li Y, Davis RL (2019) Aversive training induces both presynaptic and postsynaptic suppression in Drosophila. J Neurosci 39:9164-9172.

Zhao B, Sun J, Zhang X, Mo H, Niu Y, Li Q, Wang L, Zhong Y (2019) Long-term memory is formed immediately without the need for protein synthesis-dependent consolidation in Drosophila. Nat Commun 10:4550.

Zhao C, Widmer YF, Diegelmann S, Petrovici M, Sprecher SG, Senn W (2021) Predictive olfactory learning in Drosophila. Sci Rep 11:6795. 